\title{
Role of the microbiome in systemic therapy for pancreatic ductal adenocarcinoma (Review)
}

\author{
XING HUANG*, MAO LI*, SHENGZHONG HOU and BOLE TIAN \\ Department of Pancreatic Surgery, West China Hospital of Sichuan University, Chengdu, Sichuan 610041, P.R. China
}

Received August 3, 2021; Accepted October 18, 2021

DOI: 10.3892/ijo.2021.5281

\begin{abstract}
A large body of evidence has revealed that the microbiome serves a role in all aspects of cancer, particularly cancer treatment. To date, studies investigating the relationship between the microbiome and systemic therapy for pancreatic ductal adenocarcinoma (PDAC) are lacking. PDAC is a high-mortality malignancy (5-year survival rate; $<9 \%$ for all stages). Systemic therapy is one of the most important treatment choices for all patients; however, resistance or toxicity can affect its efficacy. Studies have supported the hypothesis that the microbiome is closely associated with the response to systemic therapy in PDAC, including the induction of drug resistance, or toxicity and therapy-related changes in microbiota composition. The present review comprehensively summarized the role of the microbiome in systemic therapy for PDAC and the associated molecular mechanisms in an attempt to provide a novel direction for the improvement of treatment response and proposed potential directions for in-depth research.
\end{abstract}

\section{Contents}

1. Introduction

2. The microbiome and PDAC systemic therapy resistance or toxicity

3. Further directions

4. Conclusion

Correspondence to: Professor Bole Tian, Department of Pancreatic Surgery, West China Hospital of Sichuan University, 37 Guoxue Alley, Chengdu, Sichuan 610041, P.R. China

E-mail: boletian0338@163.com

${ }^{*}$ Contributed equally

Key words: microbiome, pancreatic ductal adenocarcinoma, systemic therapy, resistance, toxicity

\section{Introduction}

Pancreatic ductal adenocarcinoma (PDAC) is a high-mortality malignancy with a 5-year survival rate of $9 \%$ for all stages and $\sim 90 \%$ of patients are at advanced stages exhibiting a 5-year survival rate of $3 \%$ when diagnosed (1). PDAC is expected to become the second leading cause of cancer-related mortality worldwide within the next decade, due to its gradually increasing mortality rates (2). For patients who are not screened early enough, late-stage PDAC remains difficult to treat. Systemic chemotherapy, which includes neoadjuvant therapy, adjuvant therapy and first-line or subsequent therapy, is imperative for metastatic and locally advanced PDAC, as well as for other stages of PDAC. Gemcitabine has been established as the main first-line drug for PDAC chemotherapy. Other drugs, including albumin-bound paclitaxel, 5-fluorouracil (5-FU), capecitabine, cisplatin, irinotecan, oxaliplatin and erlotinib, are used in various combinations or as monotherapy, according to disease stage and patient status (3). However, due to acquired resistance or side effects during treatment, the efficacy for patients with PDAC is not satisfactory and the 5-year survival rate has not been significantly improved.

In recent years, an association between microbiomes and the occurrence and development of PDAC have been identified. Systems biology provides a more comprehensive and multiparametric understanding of drug metabolism. The microbiome, which is the comprehensive genomic information encoded by the microbiota and its ecosystem, products and host environment, has therefore been explored as a direction for therapy (4). Although the terms 'microbiota' and 'microbiome' are used interchangeably, the microbiota should be studied more comprehensively from the perspective of omics, while the functional microbiome is indispensable $(5,6)$. Therefore, the 'microbiome' has been fully summarized and its function has been described. The composition of the microbiota is primarily determined by host genes and affected by extrinsic factors, including diet (7), antibiotics (8), surgery (9) and some drugs [e.g., proton pump inhibitors (10)]. In addition, pancreatic acini secrete peptides that can modulate the gut microbiota, relying on the $\mathrm{Ca}^{2+}$ channel calcium release-activated calcium channel protein $1(11,12)$. The specific relationship between the microbiome and cancer, including gastrointestinal (13), breast (14), liver (15), esophageal cancer (16) and PDAC (17), has attracted the attention of researchers. Specifically, a large number of clinical studies have shown that the microbiome mediates the 
response to systemic therapy and that there are therapy-related changes in microbiota composition $(18,19)$.

Initial evidence indicates that the microbiome is associated with PDAC (Table I). The microbiota can reach the pancreas via the circulatory system or the biliary/pancreatic duct, which potentially induces carcinogenesis. The human oral microbiome is a well-established independent risk factor associated with the development of PDAC (20-26), particularly Porphyromonas gingivalis, Neisseria elongate, Streptococcus mitis and Fusobacterium (17). Of note, Fusobacterium has been found to be a low-risk factor in the oral microbiota but is associated with increased cancer-specific mortality rates when present in PDAC tissue $(20,27)$. Compared with benign pancreatic neoplasms and healthy cohorts, certain gut bacteria show a differential increase in abundance in patients with PDAC $(28,29)$ and promote tumor growth in subcutaneous and liver metastasis models of PDAC by modulating immune response (30). In addition, the fecal microbiome differences between patients with PDAC and healthy subjects, or patients with pre-cancerous pancreatic lesions means that early, microbiome-based detection of PDAC is possible (31). The microbial diversity of intrapancreatic tumors in long-term survivors of PDAC is higher compared with that in short-term survivors (32); it also differed among PDAC cases with different stages of the disease (33). Mechanistically, a range of microbe-associated molecular patterns such as lipopolysaccharide (LPS), which are released by the microbiota and translocated into the pancreas, bind with selective Toll-like receptor (TLRs) and then activate signaling pathways, such as the NF- $\kappa \mathrm{B}$ and MAPK pathways, to exacerbate carcinogenesis through innate and adaptive immune suppression in PDAC $(33,34)$, which may also synergize with K-ras (35). Furthermore, the mycobiome, particularly Malassezia, may also infiltrate PDAC tissues by driving the complement cascade through mannan-binding lectin activation (36). Antibiotics targeting unique microbiota constituents administered by orogastric gavage clearly inhibits PDAC progression and enhances adaptive immunity in a model of tumor protection (33). The receptor T2R38, which could be stimulated by a bacterial-derived signaling molecule, is expressed in tumor cells in patients with PDAC, as well as in tumor-derived cell lines and could link the microbiota to cancer (37). Gut microbiota metabolism may closely regulate PDAC progression through metabolite-sensing receptors $(35,38)$. Admittedly, the underlying mechanism and link are complex, but functional research of the microbiome should improve our understanding of PDAC.

The majority of studies examining the microbiome in PDAC have focused on carcinogenicity, as the data on therapeutic action are preliminary. However, although the complex and important role of the microbiome in PDAC therapy requires in-depth study, related research is limited and the mechanisms involved remain to be fully elucidated. The aim of the present review was to outline recent microbiome research-related developments and interesting discoveries in systemic therapy drugs for PDAC and illustrate the underlying mechanisms. Promising research directions with regards to the involvement of the microbiome in PDAC treatment were also discussed and proposed.

\section{The microbiome and PDAC systemic therapy resistance or toxicity}

The majority of patients with PDAC require systemic therapy, whether that consists of surgery followed by adjuvant therapy, neoadjuvant therapy or palliative adjuvant therapy. However, since the majority of patients with PDAC develop resistance or toxicity to drugs, the treatment needs to be delivered at a lower dose or suspended before the scheduled end date, which leads to it being ineffective. Therefore, clarifying the complex mechanisms to improve treatment response is important. Functional research on the microbiota has suggested that it has the potential to induce PDAC treatment resistance or toxicity. In this section, the findings of previous studies with regards to the relationship between the microbiome and several drugs for the treatment of PDAC, including gemcitabine, 5-FU, capecitabine, oxaliplatin, irinotecan, cisplatin and erlotinib (Tables II and III), were described in detail (3).

Gemcitabine and paclitaxel. Gemcitabine has been widely used as a first-line drug for PDAC for decades (39). Several trials examining the efficacy of various drugs used either alone or in combination with gemcitabine achieved modest success, particularly nab-paclitaxel, a nanoparticle form of paclitaxel (40). Therefore, the majority of studies exploring the chemoresistance mechanisms in PDAC, including those focusing on the microbiome, focus on gemcitabine (41). Mycoplasma, which contains a number of nucleoside-metabolizing enzymes (42), could be a limiting factor for the anticancer efficiency of gemcitabine (dFdC-based chemotherapy) via cytidine deaminase (CDD), causing rapid drug catabolism in the tumor microenvironment (TME) (43). Furthermore, the deamination of gemcitabine has been shown to be indirectly potentiated by mycoplasma-derived pyrimidine nucleoside phosphorylase (PyNP) activity (43). Therefore, a CDD inhibitor can restore the activity of gemcitabine by co-administration, particularly with a thymidine phosphorylase (TP)/PyNP inhibitor (43). Geller et al (44) report that certain microbes, including Gammaproteobacteria and Mycoplasma, expressed the enzyme CDD, whose long form (CDDL) metabolizes gemcitabine into its inactive form (2',2'-difluorodeoxyuridine). In vitro, AsPC1 PDAC cells cultured with bacterial-conditioned medium are completely resistant to gemcitabine. Then, 113 human PDAC tissue samples were tested, $86(76 \%)$ of which were positive for these bacteria, particularly Gammaproteobacteria. (44) Another study demonstrates that microbes present in the gut or intratumor regions influence the response to gemcitabine (45). These studies mainly indicate that the microbiome could directly degrade gemcitabine by metabolism, which is associated with the characteristics of the drug. Despite the lack of mouse models of PDAC, these results showed that bacterial species within PDAC tissues and the TME serve an important role in gemcitabine chemoresistance.

The combination of gemcitabine and albumin-bound paclitaxel has been upgraded as a category 1 recommendation (3). Kesh et al (46) found that microbial dysbiosis increases resistance to this combination. In a pancreatic tumor-bearing mouse model of type II diabetes, Enterobacter cloacae and carbohydrate- and lipid-metabolizing bacteria are 


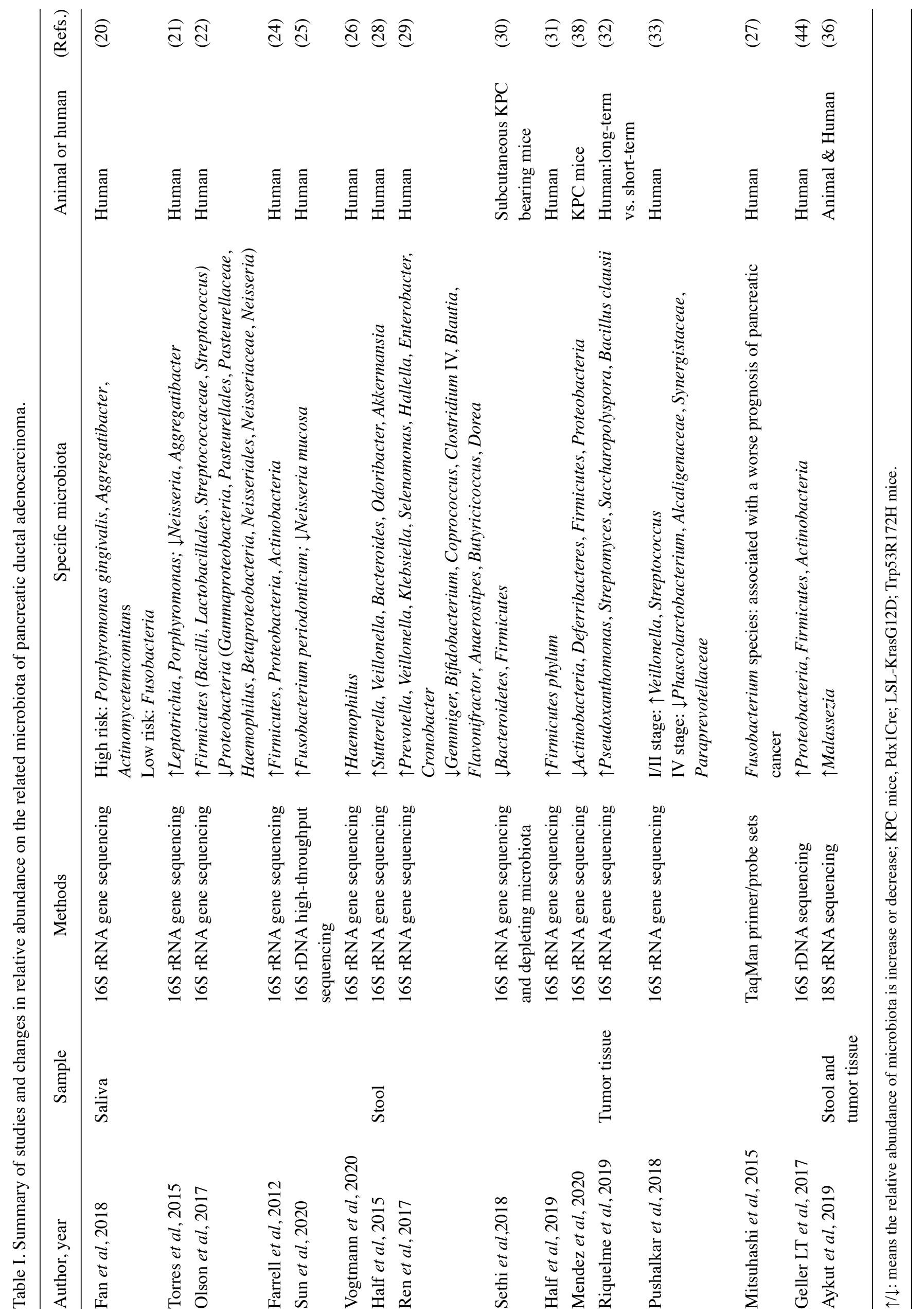




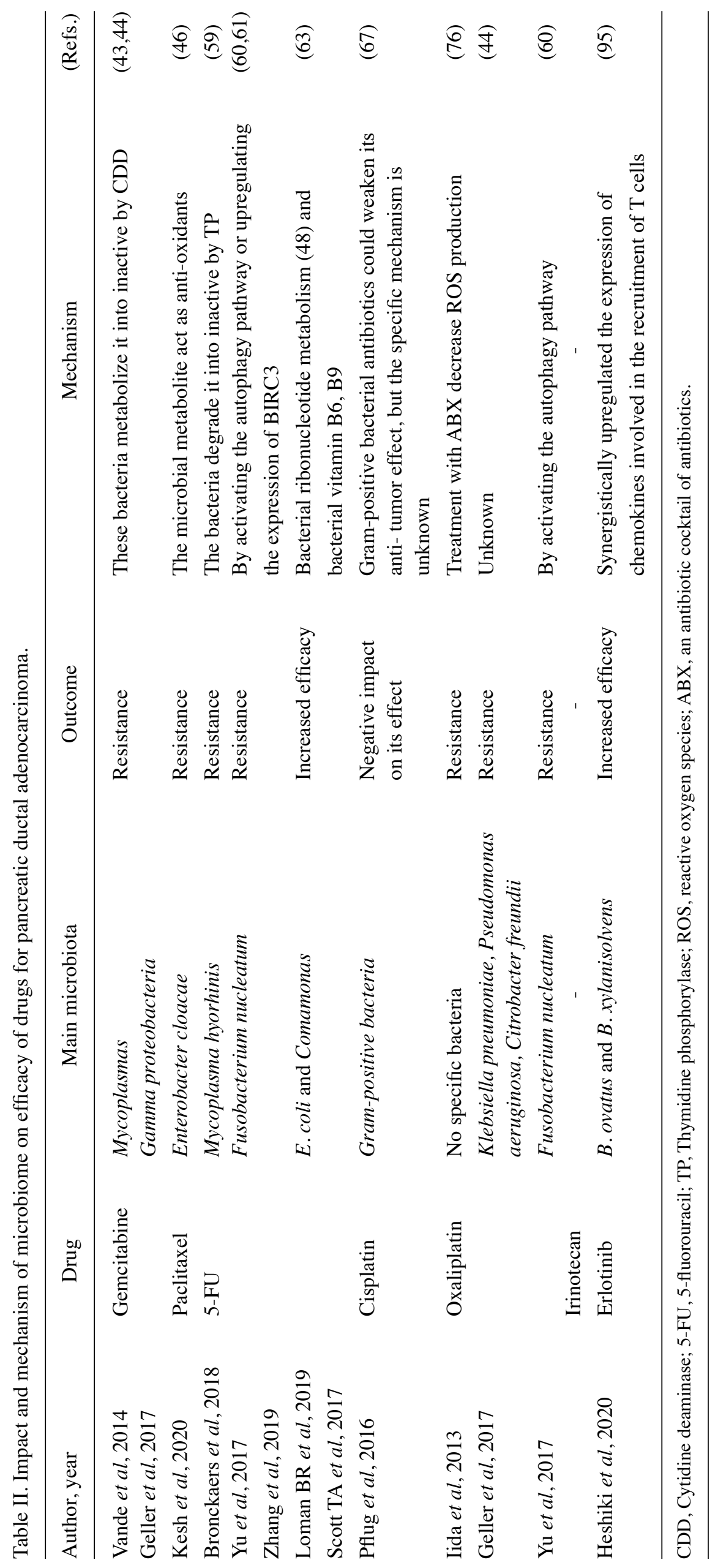




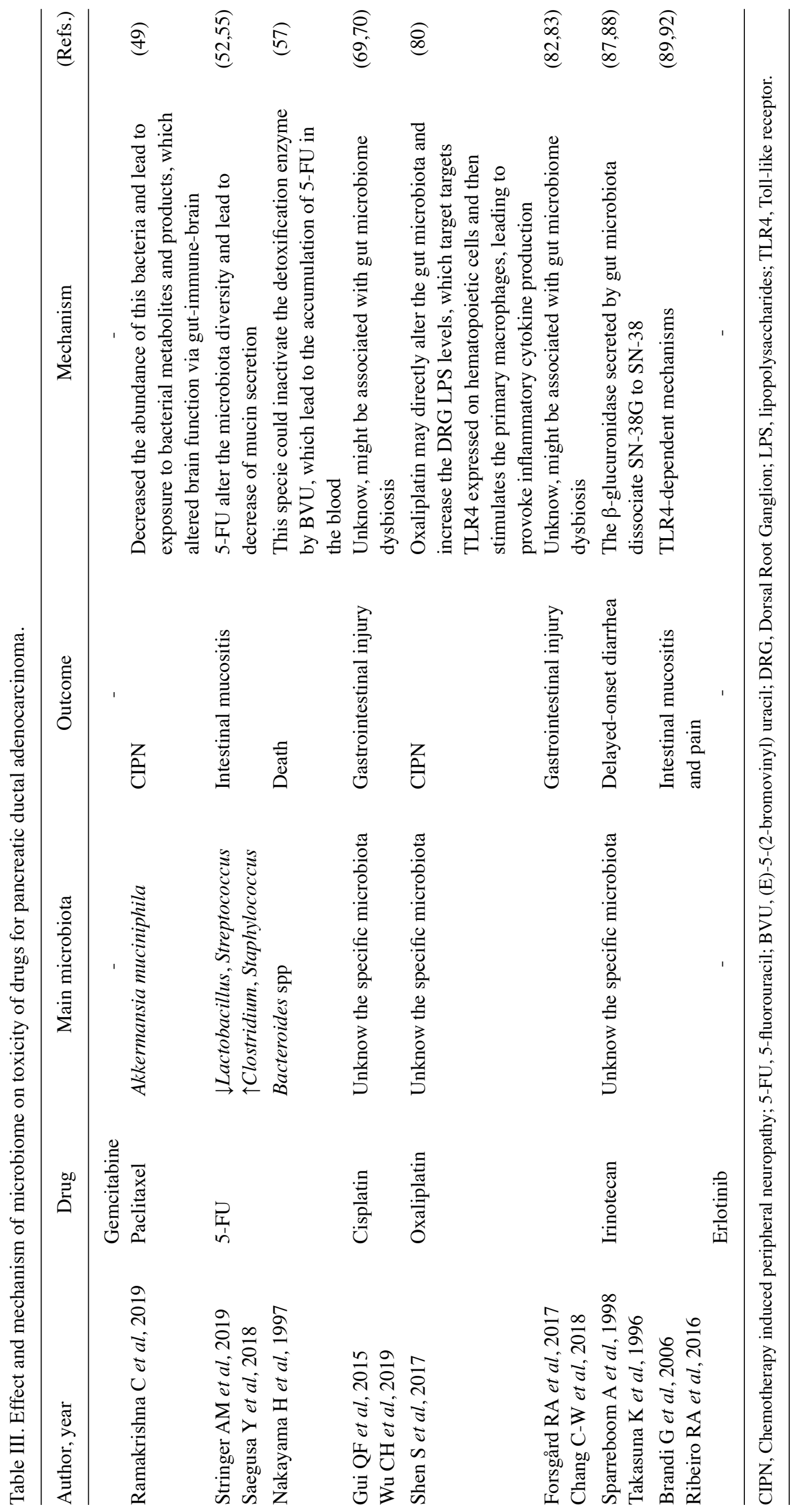


enriched. This enrichment of microbial metabolites prevents tumor cells from chemotherapy-induced accumulation of reactive oxygen species, leading to resistance (46); however, in that study, the treatment regimen was a combination of gemcitabine and paclitaxel and no study has yet focused on microbiome-induced paclitaxel monotherapy resistance. Although lactic acid bacteria, bifidobacteria and other bacteria of intestinal origin are not susceptible to paclitaxel (47), the bacterial populations are altered in paclitaxel-treated mice: butyrate-producing bacteria, including Roseburia, Eubacterium and Erysipelotrichaceae, are depleted (48) and paclitaxel treatment decreases the abundance of Akkermansia muciniphila and alters that of other bacterial taxa, which are drivers of chemotherapy-induced peripheral neuropathy (CIPN) (49). In addition, paclitaxel-containing chemotherapeutic combinations are more likely to result in Clostridioides difficile infection (50). Therefore, the antitumor effect of paclitaxel could be improved by reversing paclitaxel-induced gut microbiota dysbiosis (51).

Fluoropyrimidine. Fluoropyrimidine is also a first-line chemotherapeutic drug for patients with PDAC, including 5-FU, capecitabine and TAS-1. 5-FU is frequently administered alongside FOLFIRINOX/modified FOLFIRINOX and with or without leucovorin (3). Capecitabine, a precursor of 5-FU, can be administered alone or co-administered with gemcitabine to patients with PDAC (3). The majority of studies on 5-FU focus on its effects on the abundance of microbiota constituents and induction of mucositis (52-54). The main mechanisms of toxicity have been demonstrated. First, 5-FU alters microbiota diversity by decreasing Lactobacillus and Streptococcus abundance and increasing Clostridium and Staphylococcus abundance, leading to a decrease in the secretion of mucin, a principal factor in the physiological defense of the gastrointestinal mucosa $(52,55)$. Therefore, supplementation with the genera Lactobacillus and Bifidobacterium could protect the human gastrointestinal tract from chemotherapy (56). Secondly, the lack of a detoxification enzyme of 5-FU (hepatic dihydropyridine dehydrogenase) may lead to an increase in the systemic concentrations of 5-FU in the blood and enhanced toxicity. The gut microbiota serves a critical role in that process. Specific bacteria, including Bacteroides species (B. vulgatus, B. thetaiotaomicron, B. fragilis, B. uniformis and B. eggerthii) can hydrolyze sorivudine to (E)-5-(2-bromovinyl) uracil, which inactivates the detoxification enzyme (57).

At present, the understanding of whether the gut microbiota influences the antitumor efficacy of 5-FU treatment is limited. A previous study proposes that 5-FU together with $\mathrm{ABX}$, an antibiotic cocktail, markedly reduces the antitumor effect of 5-FU and the gut bacterial diversity and communities show significant changes compared with those after 5-FU alone or 5-FU plus probiotic treatments (58). This means that the gut flora dysbiosis contributes to the induction of 5-FU resistance. Mycoplasma hyorhinis, which was mentioned in the gemcitabine and paclitaxel section, also degrades fluoropyrimidines, including 5-FU, by TP to their inactive bases. By contrast, capecitabine, which must be metabolized to 5-fluoro-5'-deoxyuridine (5'DFUR), can benefit from TP activity (59). Fusobacterium nucleatum, an anaerobic bacterium that is parasitic in the oral cavity and highly abundant in the gut microbiota, may promote 5-FU and oxaliplatin resistance by targeting TLR4 and myeloid differentiation primary response 88 (MYD88) innate immune signaling and then downregulating the expression of microRNA (miR)-18a* and miR-4802, which activate the autophagy pathway by increasing Unc-51 like autophagy activating kinase 1 and autophagy related 7 expression (60). In addition, another study demonstrated that $F$. nucleatum confers resistance to 5-FU by upregulating the expression of baculoviral IAP repeat containing 3 via the TLR4/NF- $\mathrm{BB}$ pathway (61). These two studies suggest that fully elucidating the mechanism of the specific microbiota constituents inducing chemoresistance poses a major challenge as the same bacteria may have two or several regulatory pathways that alter drug response. García-González et al (62) found that Escherichia coli and Comamonas increase 5-FU efficacy by bacterial nucleotide metabolism and lead to the sterility of $C$. elegans, a powerful model system to study the effects of the microbiota on chemotherapeutics. In addition to this mechanism, another study reports that E. coli vitamin B6 and B9 metabolism are essential for 5-FU efficacy in the same C. elegans model (63).

Platinum salt. Cisplatin is a platinum-based potent antitumor agent used for PDAC, along with gemcitabine, but only for patients with known breast cancer type $1 / 2$ or partner and localizer of BRCA2 mutations $(3,64)$. Cisplatin causes tumor cytotoxicity by forming platinum DNA adducts and intrastrand cross-links, as well as through the modulation of the immune system $(65,66)$. Few studies have focused on microbiome-mediated cisplatin resistance $(67,68)$. Gram-positive bacterial antibiotics can weaken its antitumor effect (67), but the specific mechanism remains to be elucidated. In addition, the majority of gastrointestinal toxicities caused by cisplatin have been attributed to various events, such as oxidative stress and inflammation (68). Although there is no direct evidence that the microbiome induces toxicity, the combination of cisplatin with the commensal microbiota or agents that balance it could ameliorate cisplatin-induced gastrointestinal toxicity (69-72), as well as other adverse effects (73-75). These studies suggest the existence of a crucial intrinsic link between the microbiome and cisplatin, but additional research should focus on and clarify the mechanism.

Oxaliplatin, a third-generation platinum-based chemotherapeutic drug, has been approved for the first-line treatment of PDAC in FOLFIRINOX/modified FOLFIRINOX strategies (3). Iida et al (76) suggest that an intact commensal microbiota is indispensable for optimal responses to cancer therapy with oxaliplatin and that ABX impairs the effect of oxaliplatin by decreasing reactive oxygen species (ROS) production, which serves a crucial role in DNA damage and apoptosis (77). Although the complex microbiome holds infinite possibilities to control the response of oxaliplatin (76), the exact bacteria that serve a pivotal role are unknown. By contrast, Geller et al (44) found that certain bacteria can mediate resistance to oxaliplatin, including Klebsiella pneumoniae, Pseudomonas aeruginosa and Citrobacter freundii, but not CDDL-mediated resistance and the mechanism was unclear. In another study. Yu et al (60) demonstrate that that F. nucleatum induces oxaliplatin resistance by targeting TLR4 and MYD88 innate immune signaling and specific miRs to 
activate the autophagy pathway, similar to 5-FU. Although the microbiome can either disturb or promote the effect of oxaliplatin, it is unclear which microbes are involved or the specific mechanisms underlying their involvement.

The efficacy of oxaliplatin is limited by peripheral neuropathies, as well as gastrointestinal toxicity $(78,79)$, but whether the microbiota induces or mediates oxaliplatin toxicity has rarely been reported. Shen et al (80) reports that the gut microbiota may promote the development of oxaliplatin-induced pain, which can be reduced in germ-free mice and mice pretreated with antibiotics. Mechanistically, the dorsal root ganglion (DRG) is a key anatomical site for CIPN pathogenesis (81). Oxaliplatin may directly alter the gut microbiota and increase LPS levels in the DRG (80). LPS derived from the gut microbiota targets TLR4, which is expressed on hematopoietic cells and then stimulates primary macrophages, leading to the production of inflammatory cytokines in the DRG, such as IL-6 and TNF- $\alpha$ (80). Gastrointestinal injury is one of the toxicities induced by oxaliplatin, an effect that may be associated with alterations in the gut microbiota and activation of inflammatory processes $(82,83)$. Accordingly, fecal microbiota transplantation (FMT) can alleviate the injury (84).

Irinotecan. Although FOLFIRINOX causes marked improvements in patients with metastatic PDAC compared with gemcitabine, the $3 / 4$ toxicity rate is clearly greater (85), which always leads to a dose reduction. Irinotecan (also known as CPT-11) is the main drug in the FOLFIRINOX regimen that occasionally induces severe toxicities, which limit its use and efficacy (86). Delayed-onset diarrhea is a common clinical adverse effect. The most likely mechanism of the induction of severe diarrhea is that the $\beta$-glucuronidase secreted by the gut microbiota dissociates $\mathrm{SN}-38 \mathrm{G}$ to $\mathrm{SN}-38$, which is responsible for both antitumor activity and dose-limiting toxicity $(87,88)$. This underlying mechanism reveals that the gut microflora serves a critical role in the intestinal toxicity of irinotecan (89), even though the association appears to be controversial and mechanisms other than this enzyme, such as TLR4-dependent mechanisms (90), may be involved in irinotecan treatment $(91,92)$.

Erlotinib. Erlotinib, an EGFR tyrosine kinase inhibitor, increases overall survival when combined with gemcitabine (93) and this combination therapy is another option for patients with locally advanced or metastatic disease; it has a good performance status and is a category 1 recommendation for patients with metastatic disease in the National Comprehensive Cancer Network guidelines (3). There is little research on the relationship between the microbiome and erlotinib. Two studies indicate that certain bacteria of intestinal origin had no susceptibility to erlotinib and did not induce changes in intestinal tissue morphology, but whether there were changes in the abundance of the gut microbiome remain unknown $(47,94)$. Heshiki et al (95) found that baseline microbiota composition could predict treatment response and the responder bacteria (Bacteroides ovatus and B.xylanisolvens) increase the efficacy of erlotinib in mice more than the non-responder bacteria (Cenarchaeum symbiosum and Ruminococcus gnavus) when administered by oral gavage. Mechanistically, the responder bacteria may synergistically upregulate chemokines involved in T-cell recruitment and then enhance erlotinib efficacy (95).

\section{Further directions}

Pharmacomicrobiomics, a new discipline exploring the interactions between drugs and microbes (96), has the potential to broaden our understanding of the interplay between the microbiome and systemic therapy for PDAC. In addition, clinical metagenomic next-generation sequencing has provided a glimpse into the monitoring of chemotherapy regimens (97). In addition, the increased knowledge obtained in this field can potentially generate novel chemotherapeutic or subsequent therapy approaches to enhance efficacy and abrogate side effects by manipulating the $\alpha$ - and $\beta$-diversity of the microbiota to individualize treatment. The present review provided a detailed overview of the association between the microbiome and systemic therapy drugs for PDAC. However, since the majority of the studies' objectives are not PDAC, the evidence obtained in the present study remains limited. Therefore, carrying out research to further elucidate the role of the microbiome in PDAC systemic therapy is urgent. From the perspective of the present study, four main aspects need additional attention in future research (Fig. 1).

\section{Baseline gut microbiome for individualized chemotherapy} programs for patients with PDAC. Numerous clinical studies have investigated whether the baseline gut microbiota predicts the clinical response to systemic cancer therapy or bacterial infection (19,98-100). Aarnoutse et al (101) profile the microbiota composition before, during and after three cycles of systemic treatment with capecitabine or TAS-102 and attempt to detect a microbiota composition that predicts chemotoxicity in patients with metastatic and/or resectable colorectal cancer.

Heshiki et al (95) investigate the role of the gut microbiota in a cancer patient cohort, which comprised 26 patients with eight different types of cancer (including PDAC) treated with targeted chemotherapy $(n=15)$, or a combination of cytotoxic or targeted chemotherapy with immunotherapy $(n=11)$. Although the cancer type varies, a dendrogram shows that the cluster tends to be closely based on therapeutic effects rather than on type of cancer or therapeutic regimens (95). Based on the treatment outcome, the patients are grouped into responders and non-responders and then the differences in intestinal microbial composition and functionality are identified. Next, a classification model is built that includes species and pathways that could predict the response to anticancer treatments. In an independent validation cohort, the prediction models achieved high accuracy (area under curve $=0.75$ ) (95).

In addition, the baseline gut microbiome can also be used to predict the toxicity of chemotherapy. Stringer et al (102) analyze stool and serum samples from 26 patients with cancer receiving chemotherapy. The type of cancer and chemotherapy regimen both differed from patient to patient; the latter included capecitabine, cisplatin/5-FU, FOLFOX, 5-FU/folinic acid, COFF plus paclitaxel and carboplatin plus gemcitabine. Specific bacteria were enriched (including $E$. coli and Staphylococcus spp.) or depleted (including Lactobacillus spp., Bifidobacterium spp., Bacteroides spp. and Enterococcus spp.) in the majority of patients with chemotherapy-induced 


\section{Systemic therapy for PDAC}

(Gemcitabine, Paclitaxel, Fluoropyrimidine, Cisplatin, Oxaliplatin, Irinotecan, Erlotinib)

Multi'omics' analysis
(Metagenomics, metabonomics, proteomics, etc.)
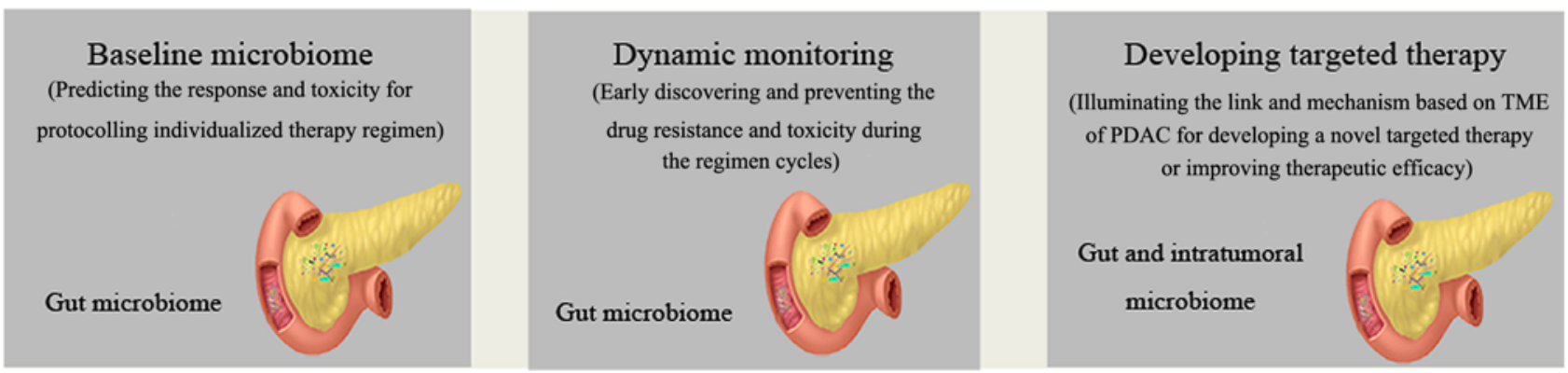
Supportive improve therapeutic methods
When unmet, precise auxiliary methods need to be taken
- Antibiotics
- Probiotics
- FMT

Figure 1. Further interrelated directions of microbiome research for improving systemic therapy for PDAC. Baseline gut microbiome sequencing and multi 'omics' functional analysis can be used to establish a prediction model for selecting a precision chemotherapy regimen. During the cycles of chemotherapy, dynamic monitoring of the changes in the gut microbiome can contribute to the early discovery of upcoming resistance or toxicity, so that certain precise interventions for balancing microbiome dysbiosis can be carried out. However, the microbiome is complex and further research should focus not only on the gut, but also on the tissue, in search of a novel targeted systemic therapy or methods for improving therapeutic efficacy. PDAC, pancreatic ductal adenocarcinoma; TME, tumor microenvironment; FMT, fecal microbiota transplantation.

diarrhea (CD) and alterations in inflammation and circulating matrix metalloproteinases were observed (102). These changes may serve as predictive biomarkers of chemotherapeutic toxicity. In addition, the biomarkers based on the baseline gut microbiome could be combined with additional biomarkers, including metabolites.

Relevant clinical studies of PDAC are not yet available. However, ongoing or previous studies have suggested that the baseline microbiota may be able to predict treatment response. Related clinical research on PDAC exploring different regimens, stages and performance statuses should therefore be performed. This research can be more comprehensive, investigating not only the gut or intratumor microbiota but also the related metabolites and other small molecules, which could be generalized to the aforementioned microbiome. That accumulated knowledge could help build a systemic and comprehensive prediction model for the response to chemotherapy regimens.

Dynamic monitoring of changes in the gut microbiome during the chemotherapy cycle and exploration of the function-mediated diversity mechanism. Systemic cancer therapies can affect the entire body, as well as the human microbiota composition and abundance, including that of gut and intratumor environment microbiota. The majority of existing studies have focused on the link between intestinal barrier dysfunction and toxicity $(44,48,52,103-108)$ In addition, whether the drugs induce changes in the microbiome and then lead to resistance is unclear. This phenomenon is called function-mediated diversity and certain studies have hinted at that possibility.

In animal studies, Lin et al (103) describe the microbiota changes during irinotecan therapy. Following irinotecan chemotherapy, cecal Clostridium cluster XI and Enterobacteriaceae, which do not mediate $\mathrm{CD}$, are increased and antimicrobial activity is excluded in vitro by irinotecan and SN-38 (103). Panebianco et al (104) demonstrate the influence of gemcitabine chemotherapy on the fecal microbiota of PC-xenografted mice. At the phylum level, Firmicutes and Bacteroidetes are considerably depleted and Proteobacteria and Verrucomicrobia are enriched. For Proteobacteria, one study demonstrates its ability to induce resistance to gemcitabine(44). At the species level,Akkermansia muciniphila and E. coli are significantly enriched, while B. acidifaciens is depleted (104). For 5-FU, the majority of studies have described the influence of chemotherapy-induced mucositis, mainly due to microbial dysbiosis, as mentioned above. A detailed study shows that the changes in jejunum, colon and fecal samples are different (52). Lactobacillus spp. and Streptococcus spp. are all depleted in the jejunum and 
colon, but Clostridium spp. are depleted and Escherichia spp. are enriched in the jejunum, while Enterococcus spp. are depleted in the colon. In the fecal samples, the results show a decreasing trend in Lactobacillus spp. and Bacteroides spp. and an increasing trend in E. coli (52). Paclitaxel-treated mice also exhibit a decrease in fecal bacterial diversity (48). Chemotherapy drugs induce changes in the microbiome not only in composition and abundance but also in the emergence of antibiotic-resistant pathogenic bacteria (105).

In a clinical study, Zwielehner et al (106) investigate the chemotherapy-induced changes in fecal microbiota. After analyzing the feces of 17 cancer patients before and after the chemotherapy cycle at four time points, the percentage of Clostridium cluster XIVa decreased from $22 \pm 13$ to $19 \pm 12 \%$, while the average proportions of Bacteroides, Bifidobacteria and Clostridium cluster IV increased (106). The changes among the four time points were also shown (106). Due to the variety of types of cancer and chemotherapy regiments, the conclusions of that study are limited and should be further confirmed in a more rigorous design. Another study identifies a significant decrease in the abundance of Firmicutes and Actinobacteria and increase in the abundance of Proteobacteria, as compared with those before chemotherapy in fecal samples (107), but the specific regimen is unknown. Kong et al (108) performed a study to identify alterations in the gut microbiota of colorectal cancer patients treated with adjuvant chemotherapy (capecitabine plus oxaliplatin) following radical surgery. Although no significant difference in the diversity and composition of the gut microbiota was observed among the various time points chemotherapy, the dynamic changes revealed by heat map clustering analysis showed that the abundance of Bacteroidetes was increased, while that of Firmicutes was decreased after chemotherapy compared to before chemotherapy (108).

These limited studies suggest that multiple cycles of chemotherapy cause changes in the gut microbiota and that altered organisms may cause drug resistance or sensitivity. This alteration in microbial diversity may be mediated and accelerated by the functional response to chemotherapy, with the microecosystems changing towards a more favorable environment (109), which has been destroyed by systemic chemotherapy. However, no related research has been conducted for PDAC. Concentrating on dynamic changes and functional response diversity will help clinicians deal with resistance or impede infection quickly and effectively.

Identifying the mechanism to develop a novel targeted therapy or improve therapeutic efficacy. The microbiota is complex and certain studies have illustrated that certain bacteria can mediate the resistance and toxicity of certain chemotherapy drugs. However, the specific mechanism may be multifactorial. For example, Fusobacterium, an oral bacterium detected in PDAC tissue $(20,27)$, mediates 5-FU resistance by activating the autophagy and TLR4/NF- $\mathrm{kB}$ pathways $(60,61)$. In addition, identifying specific bacteria and mechanisms is conducive to developing a new targeted therapy to improve resistance. For irinotecan, targeted gut microbial enzyme inhibitors may be a new potential method to alleviate gastrointestinal tract toxicity and enhance efficacy $(110,111)$. Due to the structural and functional differences in $\beta$-glucuronidases from the human gut microbes $(112,113), \beta$-glucuronidase inhibitors should be selective and not affect the survival of the microbiota $(114,115)$; therefore, the molecular mechanism should be specific. In addition, when analyzing the species and functional composition of the gut microbiome, the focus cannot only be placed on the abundant species, which do not always equate to abundant molecular functions (116). Functional analysis needs to be specific to a particular microbiome, including species with low abundance.

In addition, an increased understanding of the complex mechanisms underlying the role of the microbiome in the systemic therapy of PDAC needs to include the role of the microbiome in the TME, which is composed of a minority of malignant cells, endothelial cells, immune cells, fibroblasts and extracellular matrix (117), as well as the microbiota. Therapeutic failures of chemotherapy, particularly gemcitabine, have been attributed to the PDAC microenvironment (118). Given the complexity of the PDAC TME, the cause of resistance to chemotherapy is multifactorial and the microbiome may serve a moderate role. For example, intrapancreatic and gut-specific microbes serve as helpers in the shaping of the immunosuppressive PDAC TME, which leads to tumor-associated macrophages (TAMs) becoming highly abundant in PDAC, ranging from M1-like TAMs to immune-suppressive M2-like TAMs (33). In this process, deoxycytidine macrophages release competitively inhibits gemcitabine uptake and metabolism and leads to chemoresistance (119). Therefore, the microbiota may induce gemcitabine resistance by shaping the PDAC intratumoral immune microenvironment. The aforementioned data markedly indicate that the microbiome can mediate therapeutic responses systematically through numerous mechanisms and that these can also be structured as the 'TIMER' mechanistic framework (120). Elucidating these mechanisms will be conducive to developing a novel targeted therapy-based microbiome.

Accurately understanding the value of synergistic methods. As described aforementioned, the microbiota can provide a novel way to enhance the efficacy and reduce the toxicity of chemotherapeutic approaches. Several strategies can be used to synergize with systemic therapy to improve efficacy, such as antibiotics, probiotics, FMT, prebiotics, diet and physical activity, by modulating the composition of the microbiome $(121,122)$. However, future studies should accurately improve our understanding of the value of these synergistic methods.

Antibiotics. The use of antibiotics to remodel the diversity and constitution of the microbiota and alleviate toxicity have proven to be effective (123-125); however, they may also impair the response to chemotherapy. Iida et al (76) note that antibiotics impair oxaliplatin therapy efficacy by decreasing ROS production, which is the reason why anticancer drugs work (77) and are similarly regulated by antibiotics. The overuse of antibiotics targeting anaerobes is associated with a poor prognosis in patients with hepatocellular carcinoma who have undergone chemotherapy (126). In addition, the elimination of symbiotic bacteria increases the risk of pathogenic bacteria-induced infection (127). 
Probiotics. Probiotic supplementation is beneficial for human health (128) and can also be used as an adjuvant for cancer prevention and treatment $(129,130)$. The supernatant of Lactobacillus plantarum increases 5-FU chemosensitivity by inactivating Wnt/ $\beta$-catenin signaling (131). Lactobacillus also enhances the effects of cisplatin by upregulating interferon- $\gamma$, granzyme B and perforin-1 expression (69) and Lactobacillus rhamnosus can prevent 5-FU/oxaliplatin-induced intestinal injury $(53,132)$. Irinotecan-induced intestinal injury can also be prevented by pretreatment with bacteria (133). Certain clinical studies show that combined probiotics reduce the frequency of gastrointestinal complaints during chemotherapy cycles $(134,135)$.

In addition to probiotics, other combinations should be explored, such as metabolites or digestive enzymes. Identifying an improved combination of probiotics can significantly reduce the untoward effects of chemotherapy (136). Urolithin A, which is the main metabolite produced by the human gut microbiota, can potentiate the effects of both 5-FU and 5-dFUR on colon cancer cells (137). Probiotics supplemented with digestive enzymes can restore the gut microbial community and protect against 5-FU-induced gut dysbiosis (56).

FMT. There are few studies on FMT for improving systemic anticancer therapy. Le Bastard et al (138) assess the efficacy of FMT in 5-FU-induced gut dysbiosis in a mouse model. FMT ameliorates the disruption of the intestinal microbiota by significantly enriching the species with anti-inflammatory properties in mice (138). The results show that FMT has the potential to improve the resistance and toxicity induced by systemic therapy for PDAC. However, due to its uncertainties, FMT might increase the chance of infection and fecal donor selection and screening are difficult. Therefore, selective microbiota transplantation may be a better choice and additional studies should be carried out to investigate that option.

\section{Conclusion}

Although few of these studies have focused on PDAC, the mechanism underlying drug alterations by the microbiome may be similar. Microbiome studies provide a novel direction for the improvement of the response to systemic therapy for PDAC. A deep exploration of the mechanism and the relationship between the microbiome and systemic therapy drugs for PDAC is essential, due to the low survival rate and chemotherapeutic resistance of PDAC. In clinical practice, the combination of the microbiota and its metabolites and metabolic pathways could be used to establish a model for predicting the response to systemic chemotherapy regimens, which can be conducted flexibly and individually. During regimen cycles, the microbiota is destroyed, inducing resistance. Therefore, dynamic monitoring of the gut microbiota and timely adjustment of the regimen or restoration of the composition of the microbiome through the use of cooperative strategies may prove beneficial. Admittedly, the model and detection of the microbiome composition of patients should be fast, robust and inexpensive. In addition, mechanistic studies of the microbiome could provide novel targeted therapies or synergetic schemes to establish personalized medicine for each patient. In conclusion, the relationship and main mechanism between the microbiome and drugs for PDAC treatment were outlined in the present review and certain directions for future research were proposed.

\section{Acknowledgements}

Not applicable.

\section{Funding}

This study was supported by Sichuan Science and Technology Program (grant no. 2021YFS0234).

\section{Availability of data and materials}

Data sharing is not applicable to this article, as no data sets were generated or analyzed during the current study.

\section{Authors' contributions}

BT conceived and supervised the work. $\mathrm{XH}, \mathrm{ML}$ and $\mathrm{SH}$ researched data and contributed equally to discussion of content, XH and ML wrote the manuscript. All authors reviewed and approved the final manuscript.

\section{Ethics approval and consent to participate}

Not applicable.

\section{Patient consent for publication}

Not applicable.

\section{Competing interests}

The authors declare that they have no competing interests

\section{References}

1. Siegel RL, Miller KD and Jemal A: Cancer statistics, 2019. CA Cancer J Clin 69: 7-34, 2019.

2. Rahib L, Smith BD, Aizenberg R, Rosenzweig AB, Fleshman JM and Matrisian LM: Projecting cancer incidence and deaths to 2030: The unexpected burden of thyroid, liver, and pancreas cancers in the United States. Cancer Res 74: 2913-2921, 2014

3. Network (NCCN): NCC: Clinical Practice Guidelines in Oncology. Pancreatic Adenocarcinoma, Version 1. NCCN, Pennsylvania, 2020. https://www.nccn.org/professionals/physician_gls/pdf/ pancreatic.pdf. Accessed November 26, 2019.

4. Marchesi JR, Adams DH, Fava F, Hermes GDA, Hirschfield GM, Hold G, Quraishi MN, Kinross J, Smidt H, Tuohy KM, et al: The gut microbiota and host health: A new clinical frontier. Gut 65: 330-339, 2016.

5. Heintz-Buschart A and Wilmes P: Human gut microbiome: Function matters. Trends Microbiol 26: 563-574, 2018.

6. Human Microbiome Project Consortium: Structure, function and diversity of the healthy human microbiome. Nature 486: 207-214, 2012.

7. Kolodziejczyk AA, Zheng D and Elinav E: Diet-microbiota interactions and personalized nutrition. Nat Rev Microbiol 17: 742-753, 2019.

8. Ianiro G, Tilg H and Gasbarrini A: Antibiotics as deep modulators of gut microbiota: Between good and evil. Gut 65: 1906-1915, 2016.

9. Guyton K and Alverdy JC: The gut microbiota and gastrointestinal surgery. Nat Rev Gastroenterol Hepatol 14: 43-54, 2017. 
10. Imhann F, Bonder MJ, Vila AV, Fu J, Mujagic Z, Vork L, Tigchelaar EF, Jankipersadsing SA, Cenit MC, Harmsen HJ, et al: Proton pump inhibitors affect the gut microbiome. Gut 65: 740-748, 2016.

11. Tilg $\mathrm{H}$ and Adolph TE: Beyond digestion: The pancreas shapes intestinal microbiota and immunity. Cell Metab 25: 495-496, 2017.

12. Ahuja M, Schwartz DM, Tandon M, Son A, Zeng M, Swaim W, Eckhaus M, Hoffman V, Cui Y, Xiao B, et al: Orail-mediated antimicrobial secretion from pancreatic acini shapes the gut microbiome and regulates gut innate immunity. Cell Metab 25 635-646, 2017.

13. Panebianco C, Potenza A, Andriulli A and Pazienza V: Exploring the microbiota to better understand gastrointestinal cancers physiology. Clin Chem Lab Med 56: 1400-1412, 2018.

14. Balhouse BN, Patterson L, Schmelz EM, Slade DJ and Verbridge SS: N-(3-oxododecanoyl)-L-homoserine lactone interactions in the breast tumor microenvironment: Implications for breast cancer viability and proliferation in vitro. PLoS One 12 : e0180372, 2017.

15. Grąt M, Wronka KM, Krasnodębski M, Masior L, Lewandowski Z, Kosińska I, Grąt K, Stypułkowski J, Rejowski S, Wasilewicz M, et al: Profile of gut microbiota associated with the presence of hepatocellular cancer in patients with liver cirrhosis. Transplant Proc 48: 1687-1691, 2016.

16. Zaidi AH, Kelly LA, Kreft RE, Barlek M, Omstead AN, Matsui D, Boyd NH, Gazarik KE, Heit MI, Nistico L, et al: Associations of microbiota and toll-like receptor signaling pathway in esophageal adenocarcinoma. BMC Cancer 16: 52, 2016.

17. Wei MY, Shi S, Liang C, Meng QC, Hua J, Zhang YY, Liu J, Zhang $\mathrm{B}, \mathrm{Xu} \mathrm{J}$ and $\mathrm{Yu} \mathrm{XJ}$ : The microbiota and microbiome in pancreatic cancer: More influential than expected. Mol Cancer 18: 97, 2019

18. Roy S and Trinchieri G: Microbiota: A key orchestrator of cancer therapy. Nat Rev Cancer 17: 271-285, 2017.

19. Aarnoutse R, Ziemons J, Penders J, Rensen SS, de Vos-Geelen J and Smidt ML: The clinical link between human intestinal microbiota and systemic cancer therapy. Int J Mol Sci 20: 4145, 2019.

20. Fan X, Alekseyenko AV, Wu J, Peters BA, Jacobs EJ, Gapstur SM, Purdue MP, Abnet CC, Stolzenberg-Solomon R, Miller G, et al: Human oral microbiome and prospective risk for pancreatic cancer: A population-based nested case-control study. Gut 67 : 120-127, 2018.

21. Torres PJ, Fletcher EM, Gibbons SM, Bouvet M, Doran KS and Kelley ST: Characterization of the salivary microbiome in patients with pancreatic cancer. PeerJ 3: e1373, 2015.

22. Olson SH, Satagopan J, Xu Y, Ling L, Leong S, Orlow I, Saldia A, Li P, Nunes P, Madonia V, et al: The oral microbiota in patients with pancreatic cancer, patients with IPMNs, and controls: A pilot study. Cancer Causes Control 28: 959-969, 2017.

23. Michaud DS, Izard J, Wilhelm-Benartzi CS, You DH, Grote VA, Tjønneland A, Dahm CC, Overvad K, Jenab M, Fedirko V, et al: Plasma antibodies to oral bacteria and risk of pancreatic cancer in a large European prospective cohort study. Gut 62: 1764-1770, 2013.

24. Farrell JJ, Zhang L, Zhou H, Chia D, Elashoff D, Akin D, Paster BJ, Joshipura K and Wong DT: Variations of oral microbiota are associated with pancreatic diseases including pancreatic cancer. Gut 61: 582-588, 2012.

25. Sun H, Zhao X, Zhou Y, Wang J, Ma R, Ren X, Wang H and Zou L: Characterization of oral microbiome and exploration of potential biomarkers in patients with pancreatic cancer. Biomed Res Int 2020: 4712498, 2020.

26. VogtmannE,Han Y,Caporaso JG, Bokulich N, Mohamadkhani A, Moayyedkazemi A, Hua X, Kamangar F, Wan Y, Suman S, et al: Oral microbial community composition is associated with pancreatic cancer: A case-control study in Iran. Cancer Med 9: 797-806, 2020

27. Mitsuhashi K, Nosho K, Sukawa Y, Matsunaga Y, Ito M, Kurihara H, Kanno S, Igarashi H, Naito T, Adachi Y, et al: Association of Fusobacterium species in pancreatic cancer tissues with molecular features and prognosis. Oncotarget 6 : 7209-7220, 2015.

28. Half E, Keren N, Dorfman T, Reshef L, Lachter I, Kluger Y, Konikoff $F$ and Gphna U: Specific changes in fecal microbiota may differentiate Pancreatic Cancer patients from healthy individuals. Ann Oncol 26: iv48, 2015.

29. Ren Z: Gut microbial profile analysis by MiSeq sequencing of pancreatic carcinoma patients in China. Oncotarget 8: 95176-95191, 2017
30. Sethi V, Kurtom S, Tarique M, Lavania S, Malchiodi Z, Hellmund L, Zhang L, Sharma U, Giri B, Garg B, et al: Gut microbiota promotes tumor growth in mice by modulating immune response. Gastroenterology 155: 33-37.e36, 2018.

31. Half E, Keren N, Reshef L, Dorfman T, Lachter I, Kluger Y, Reshef N, Knobler H, Maor Y, Stein A, et al: Fecal microbiome signatures of pancreatic cancer patients. Sci Rep 9: 16801, 2019.

32. Riquelme E, Zhang Y, Zhang L, Montiel M, Zoltan M, Dong W, Quesada P, Sahin I, Chandra V, Lucas AS, et al: Tumor microbiome diversity and composition influence pancreatic cancer outcomes. Cell 178: 795-806 e712, 2019.

33. Pushalkar S, Hundeyin M, Daley D, Zambirinis CP, Kurz E, Mishra A, Mohan N, Aykut B, Usyk M, Torres LE, et al: The pancreatic cancer microbiome promotes oncogenesis by induction of innate and adaptive immune suppression. Cancer Discov 8: 403-416, 2018.

34. Ikebe M,Kitaura Y,Nakamura M, Tanaka H, Yamasaki A, Nagai S, Wada J, Yanai K, Koga K, Sato N, et al: Lipopolysaccharide (LPS) increases the invasive ability of pancreatic cancer cells through the TLR4/MyD88 signaling pathway. J Surg Oncol 100: 725-731, 2009.

35. Eibl G and Rozengurt E: KRAS, YAP, and obesity in pancreatic cancer: A signaling network with multiple loops. Semin Cancer Biol 54: 50-62, 2019.

36. Aykut B, Pushalkar S, Chen R, Li Q, Abengozar R, Kim JI, Shadaloey SA, Wu D, Preiss P, Verma N, et al: The fungal mycobiome promotes pancreatic oncogenesis via activation of MBL. Nature 574: 264-267, 2019.

37. Gaida MM, Mayer C, Dapunt U, Stegmaier S, Schirmacher P, Wabnitz GH and Hänsch GM: Expression of the bitter receptor T2R38 in pancreatic cancer: Localization in lipid droplets and activation by a bacteria-derived quorum-sensing molecule. Oncotarget 7: 12623-12632, 2016.

38. Mendez R, Kesh K, Arora N, Martino LD, McAllister F, Merchant N, Banerjee S and Banerjee S: Microbial dysbiosis and polyamine metabolism as predictive markers for early detection of pancreatic cancer. Carcinogenesis 41: 561-570, 2020.

39. Burris HA III, Moore MJ, Andersen J, Green MR, Rothenberg ML, Modiano MR, Cripps MC, Portenoy RK, Storniolo AM, Tarassoff P, et al: Improvements in survival and clinical benefit with gemcitabine as first-line therapy for patients with advanced pancreas cancer: A randomized trial. J Clin Oncol 15: 2403-2413, 1997.

40. Von Hoff DD, Ramanathan RK, Borad MJ, Laheru DA, Smith LS Wood TE, Korn RL, Desai N, Trieu V, Iglesias JL, et al: Gemcitabine plus nab-paclitaxel is an active regimen in patients with advanced pancreatic cancer: A phase I/II trial. J Clin Oncol 29: 4548-4554, 2011

41. Gnanamony $\mathrm{M}$ and Gondi CS: Chemoresistance in pancreatic cancer: Emerging concepts. Oncol Lett 13: 2507-2513, 2017.

42. Neale GA, Mitchell A and Finch LR: Enzymes of pyrimidine deoxyribonucleotide metabolism in Mycoplasma mycoides subsp. Mycoides. J Bacteriol 156: 1001-1005, 1983.

43. Voorde JV, Sabuncuoğlu S, Noppen S, Hofer A, Ranjbarian F, Fieuws S, Balzarini J and Liekens S: Nucleoside-catabolizing enzymes in mycoplasma-infected tumor cell cultures compromise the cytostatic activity of the anticancer drug gemcitabine. J Biol Chem 289: 13054-13065, 2014.

44. Geller LT, Barzily-Rokni M, Danino T, Jonas OH, Shental N, Nejman D, Gavert N, Zwang Y, Cooper ZA, Shee K, et al: Potential role of intratumor bacteria in mediating tumor resistance to the chemotherapeutic drug gemcitabine. Science 357: 1156-1160, 2017

45. Lehouritis P, Cummins J, Stanton M, Murphy CT, McCarthy FO, Reid G, Urbaniak C, Byrne WL and Tangney M: Local bacteria affect the efficacy of chemotherapeutic drugs. Sci Rep 5: 14554, 2015.

46. Kesh K, Mendez R, Abdelrahman L, Banerjee S and Banerjee S: Type 2 diabetes induced microbiome dysbiosis is associated with therapy resistance in pancreatic adenocarcinoma. Microb Cell Fact 19: 75, 2020.

47. Florez AB, Sierra M, Ruas-Madiedo P and Mayo B: Susceptibility of lactic acid bacteria, bifidobacteria and other bacteria of intestinal origin to chemotherapeutic agents. Int J Antimicrob Agents 48: 547-550, 2016.

48. Loman BR, Jordan KR, Haynes B, Bailey MT and Pyter LM: Chemotherapy-induced neuroinflammation is associated with disrupted colonic and bacterial homeostasis in female mice. Sci Rep 9: 16490-16490, 2019. 
49. Ramakrishna C, Corleto J, Ruegger PM, Logan GD, Peacock BB Mendonca S, Yamaki S, Adamson T, Ermel R, McKemy D, et al: Dominant role of the gut microbiota in chemotherapy induced neuropathic pain. Sci Rep 9: 20324-20324, 2019.

50. Peretz A, Shlomo IB, Nitzan O, Bonavina L, Schaffer PM and Schaffer M: Clostridium difficile Infection: Associations with chemotherapy, radiation therapy, and targeting therapy treatments. Curr Med Chem 23: 4442-4449, 2016.

51. Su J, Li D, Chen Q, Li M, Su L, Luo T, Liang D, Lai G, Shuai O, Jiao C, et al: Anti-breast cancer enhancement of a polysaccharide from spore of ganoderma lucidum with paclitaxel: Suppression on tumor metabolism with gut microbiota reshaping. Front Microbiol 9: 3099, 2018.

52. Stringer AM, Gibson RJ, Logan RM, Bowen JM, Yeoh AS, Hamilton $\mathbf{J}$ and Keefe DM: Gastrointestinal microflora and mucins may play a critical role in the development of 5-fluorouracil-induced gastrointestinal mucositis. Exp Biol Med (Maywood) 234: 430-441, 2009.

53. Yeung CY, Chiau JS, Cheng ML, Chan WT, Chang SW, Chang YH, Jiang CB and Lee HC: Modulations of probiotics on gut microbiota in a 5-fluorouracil-induced mouse model of mucositis. J Gastroenterol Hepatol 35: 806-814, 2020.

54. Vanlancker E, Vanhoecke B, Smet R, Props R and Van de Wiele T: 5-Fluorouracil sensitivity varies among oral micro-organisms. J Med Microbiol 65: 775-783, 2016.

55. Saegusa Y, Ichikawa T, Iwai T, Goso Y, Okayasu I, Ikezawa T, Shikama N, Saigenji K and Ishihara K: Changes in the mucus barrier of the rat during 5-fluorouracil-induced gastrointestinal mucositis. Scand J Gastroenterol 43: 59-65, 2008.

56. Ichim TE, Kesari S and Shafer K: Protection from chemotherapy- and antibiotic-mediated dysbiosis of the gut microbiota by a probiotic with digestive enzymes supplement. Oncotarget 9 : 30919-30935, 2018.

57. Nakayama H, Kinouchi T, Kataoka K, Akimoto S, Matsuda $Y$ and Ohnishi Y: Intestinal anaerobic bacteria hydrolyse sorivudine, producing the high blood concentration of 5-(E)-(2-bromovinyl) uracil that increases the level and toxicity of 5-fluorouracil. Pharmacogenetics 7: 35-43, 1997.

58. Yuan L, Zhang S, Li H, Yang F, Mushtaq N, Ullah S, Shi Y, An $\mathrm{C}$ and $\mathrm{Xu} \mathrm{J}$ : The influence of gut microbiota dysbiosis to the efficacy of 5-Fluorouracil treatment on colorectal cancer. Biomed Pharmacother 108: 184-193, 2018.

59. Bronckaers A, Balzarini J and Liekens S: The cytostatic activity of pyrimidine nucleosides is strongly modulated by Mycoplasma hyorhinis infection: Implications for cancer therapy. Biochem Pharmacol 76: 188-197, 2008.

60. Yu T, Guo F, Yu Y, Sun T, Ma D, Han J, Qian Y, Kryczek I, Sun D, Nagarsheth N, et al: Fusobacterium nucleatum promotes chemoresistance to colorectal cancer by modulating autophagy. Cell 170: 548-563.e516, 2017.

61. Zhang S, Yang Y, Weng W, Guo B, Cai G, Ma Y and Cai S Fusobacterium nucleatum promotes chemoresistance to 5 -fluorouracil by upregulation of BIRC3 expression in colorectal cancer. J Exp Clin Cancer Res 38: 14, 2019.

62. García-González AP, Ritter AD, Shrestha S, Andersen EC, Yilmaz LS and Walhout AJM: Bacterial metabolism affects the C. Elegans response to cancer chemotherapeutics. Cell 169: 431-441, 2017.

63. Scott TA, Quintaneiro LM, Norvaisas P, Lui PP, Wilson MP, Leung KY, Herrera-Dominguez L, Sudiwala S, Pessia A, Clayton PT, et al: Host-microbe co-metabolism dictates cancer drug efficacy in C. Elegans. Cell 169: 442-456.e418, 2017.

64. Fogelman D, Sugar EA, Oliver G, Shah N, Klein A, Alewine C, Wang H, Javle M, Shroff R, Wolff RA, et al: Family history as a marker of platinum sensitivity in pancreatic adenocarcinoma. Cancer Chemother Pharmacol 76: 489-498, 2015.

65. Siddik ZH: Cisplatin: Mode of cytotoxic action and molecular basis of resistance. Oncogene 22: 7265-7279, 2003.

66. Hato SV, Khong A, de Vries IJM and Lesterhuis WJ: Molecular pathways: The immunogenic effects of platinum-based chemotherapeutics. Clin Cancer Res 20: 2831-2837, 2014.

67. Pflug N, Kluth S, Vehreschild JJ, Bahlo J, Tacke D, Biehl L, Eichhorst B, Fischer K, Cramer P, Fink AM, et al: Efficacy of antineoplastic treatment is associated with the use of antibiotics that modulate intestinal microbiota. Oncoimmunology 5: e1150399, 2016.

68. Shahid F, Farooqui Z and Khan F: Cisplatin-induced gastrointestinal toxicity: An update on possible mechanisms and on available gastroprotective strategies. Eur J Pharmacol 827: 49-57, 2018.
69. Gui QF, Lu HF, Zhang CX, Xu ZR and Yang YH: Well-balanced commensal microbiota contributes to anti-cancer response in a lung cancer mouse model. Genet Mol Res 14: 5642-5651, 2015.

70. Wu CH, Ko JL, Liao JM, Huang SS, Lin MY, Lee LH, Chang LY and $\mathrm{Ou} C \mathrm{C}$ : D-methionine alleviates cisplatin-induced mucositis by restoring the gut microbiota structure and improving intestinal inflammation. Ther Adv Med Oncol 11: $1758835918821021,2019$.

71. Feng X, Cheng Q, Meng Q, Yang Y and Nie K: Effects of ondansetron and [6]-gingerol on pica and gut microbiota in rats treated with cisplatin. Drug Des Devel Ther 13: 2633-2641, 2019.

72. Zhou P, Li Z, Xu D, Wang Y, Bai Q, Feng Y, Su G, Chen P, Wang Y, Liu H, et al: Cepharanthine hydrochloride improves cisplatin chemotherapy and enhances immunity by regulating intestinal microbes in mice. Front Cell Infect Microbiol 9: 225: doi: 10.3389, 2019.

73. Lee TH, Park D, Kim YJ, Lee I, Kim S, Oh CT, Kim JY, Yang J and Jo SK: Lactobacillus salivarius BP121 prevents cisplatin-induced acute kidney injury by inhibition of uremic toxins such as indoxyl sulfate and p-cresol sulfate via alleviating dysbiosis. Int J Mol Med 45: 1130-1140, 2020.

74. Lee YJ, Li KY, Wang PJ, Huang HW and Chen MJ: Alleviating chronic kidney disease progression through modulating the critical genus of gut microbiota in a cisplatin-induced Lanyu pig model. J Food Drug Anal 28: 103-114, 2020.

75. Zhao L, Xing C, Sun W, Hou G, Yang G and Yuan L: Lactobacillus supplementation prevents cisplatin-induced cardiotoxicity possibly by inflammation inhibition. Cancer Chemother Pharmacol 82: 999-1008, 2018.

76. Iida N, Dzutsev A, Stewart CA, Smith L, Bouladoux N, Weingarten RA, Molina DA, Salcedo R, Back T, Cramer S, et al: Commensal bacteria control cancer response to therapy by modulating the tumor microenvironment. Science 342: 967-970, 2013.

77. Ozben T: Oxidative stress and apoptosis: Impact on cancer therapy. J Pharm Sci 96: 2181-2196, 2007.

78. Ewertz M, Qvortrup C and Eckhoff L: Chemotherapy-induced peripheral neuropathy in patients treated with taxanes and platinum derivatives. Acta Oncol 54: 587-591, 2015.

79. Stojanovska V, Sakkal S and Nurgali K: Platinum-based chemotherapy: Gastrointestinal immunomodulation and enteric nervous system toxicity. Am J Physiol Gastrointest Liver Physiol 308: G223-G232, 2015 .

80. Shen S, Lim G, You Z, Ding W, Huang P, Ran C, Doheny J, Caravan J, Tate S, Hu K, et al: Gut microbiota is critical for the induction of chemotherapy-induced pain. Nat Neurosci 20: 1213-1216, 2017.

81. Sprowl JA, Ciarimboli G, Lancaster CS, Giovinazzo H, Gibson AA, Du G, Janke LJ, Cavaletti G, Shields AF and Sparreboom A: Oxaliplatin-induced neurotoxicity is dependent on the organic cation transporter OCT2. Proc Natl Acad Sci USA 110: 11199-11204, 2013.

82. Forsgård RA, Marrachelli VG, Korpela K, Frias R, Collado MC, Korpela R, Monleon D, Spillmann T and Österlund P: Chemotherapy-induced gastrointestinal toxicity is associated with changes in serum and urine metabolome and fecal microbiota in male Sprague-Dawley rats. Cancer Chemother Pharmacol 80: 317-332, 2017.

83. Chang CW, Liu CY, Lee HC, Huang YH, Li LH, Chiau JS, Wang TE, Chu CH, Shih SC, Tsai TH and Chen YJ: Variety probiotic preventively attenuates 5-fluorouracil/oxaliplatininduced intestinal injury in a syngeneic colorectal cancer model. Front Microbiol 9: 983, 2018.

84. Chang CW, Lee HC, Li LH, Chiau JS, Wang TE, Chuang WH, Chen MJ, Wang HY, Shih SC, Liu CY, et al: Fecal microbiota transplantation prevents intestinal injury, upregulation of toll-like receptors, and 5-fluorouracil/oxaliplatin-induced toxicity in colorectal cancer. Int J Mol Sci 21: 386, 2020.

85. Conroy T, Desseigne F, Ychou M, Bouché O, Guimbaud R, Bécouarn Y, Adenis A, Raoul JL, Gourgou-Bourgade S, de la Fouchardière $\mathrm{C}$, et al: FOLFIRINOX versus gemcitabine for metastatic pancreatic cancer. N Engl J Med 364: 1817-1825, 2011.

86. Vanhoefer U, Harstrick A, Achterrath W, Cao S, Seeber S and Rustum YM: Irinotecan in the treatment of colorectal cancer: Clinical overview. J Clin Oncol 19: 1501-1518, 2001.

87. Sparreboom A, de Jonge MJ, de Bruijn P, Brouwer E, Nooter K, Loos WJ, van Alphen RJ, Mathijssen RH, Stoter G and Verweij J: Irinotecan (CPT-11) metabolism and disposition in cancer patients. Clin Cancer Res 4: 2747-2754, 1998. 
88. Takasuna K, Hagiwara T, Hirohashi M, Kato M, Nomura M, Nagai E, Yokoi $\mathrm{T}$ and Kamataki T: Involvement of beta-glucuronidase in intestinal microflora in the intestinal toxicity of the antitumor camptothecin derivative irinotecan hydrochloride (CPT-11) in rats. Cancer Res 56: 3752-3757, 1996.

89. Brandi G, Dabard J, Raibaud P, Battista MD, Bridonneau C, Pisi AM, Labate AM, Pantaleo MA, Vivo AD and Biasco G: Intestinal microflora and digestive toxicity of irinotecan in mice. Clin Cancer Res 12: 1299-1307, 2006.

90. Wardill HR, Gibson RJ, Van Sebille YZA, Secombe KR, Coller JK, White IA, Manavis J, Hutchinson MR, Staikopoulos V, Logan RM and Bowen JM: Irinotecan-induced gastrointestinal dysfunction and pain are mediated by common TLR4-dependent mechanisms. Mol Cancer Ther 15: 1376-1386, 2016.

91. Pedroso SHSP, Vieira AT, Bastos RW, Oliveira JS, Cartelle CT, Arantes RM, Soares PM, Generoso SV, Cardoso VN, Teixeira MM, et al: Evaluation of mucositis induced by irinotecan after microbial colonization in germ-free mice. Microbiology 161: 1950-1960, 2015.

92. Ribeiro RA, Wanderley CWS, Wong DVT, Mota JM, Leite CA, Souza MH, Cunha FQ and Lima-Júnior RC: Irinotecan- and 5-fluorouracil-induced intestinal mucositis: Insights into pathogenesis and therapeutic perspectives. Cancer Chemother Pharmacol 78: 881-893, 2016.

93. Moore MJ, Goldstein D, Hamm J, Figer A, Hecht JR, Gallinger S, Au HJ, Murawa P, Walde D, Wolff RA, et al: Erlotinib plus gemcitabine compared with gemcitabine alone in patients with advanced pancreatic cancer: A phase III trial of the national cancer institute of Canada clinical trials group. J Clin Oncol 25: 1960-1966, 2007.

94. Forsgård RA, Marrachelli VG, Lindén J, Frias R, Collado MC, Korpela R, Monleon D, Spillmann T and Österlund P: Two-week aflibercept or erlotinib administration does not induce changes in intestinal morphology in male sprague-dawley rats but aflibercept affects serum and urine metabolic profiles. Transl Oncol 12: 1122-1130, 2019.

95. Heshiki Y, Vazquez-Uribe R, Li J, Ni Y, Quainoo S, Imamovic L, Li J, Sørensen M, Chow BK, Weiss GJ, et al: Predictable modulation of cancer treatment outcomes by the gut microbiota. Microbiome 8: 28, 2020

96. Panebianco C, Andriulli A and Pazienza V: Pharmacomicrobiomics: Exploiting the drug-microbiota interactions in anticancer therapies. Microbiome 6: 92, 2018.

97. Chiu CY and Miller SA: Clinical metagenomics. Net Rev Genet 20: 341-355, 2019.

98. Chaput N, Lepage P, Coutzac C, Soularue E, Roux KL, Monot C, Boselli L, Routier E, Cassard L, Collins M, et al: Baseline gut microbiota predicts clinical response and colitis in metastatic melanoma patients treated with ipilimumab. Ann Oncol 28 : 1368-1379, 2017.

99. Farowski F, Solbach P, Tsakmaklis A, Brodesser S, Aguilar MR, Cornely OA, Dettmer K, Higgins PG, Suerbaum S, Jazmati N, et al: Potential biomarkers to predict outcome of faecal microbiota transfer for recurrent Clostridioides difficile infection. Dig Liver Dis 51: 944-951, 2019.

100. Khanna S, Montassier E, Schmidt B, Patel R, Knights D, Pardi DS and Kashyap P: Gut microbiome predictors of treatment response and recurrence in primary clostridium difficile infection. Aliment Pharmacol Ther 44: 715-727, 2016.

101. Aarnoutse R, de Vos-Geelen JMPGM, Penders J, Boerma EG, Warmerdam FA, Goorts B, Damink SWM, Soons Z, Rensen SS and Smidt ML: Study protocol on the role of intestinal microbiota in colorectal cancer treatment: A pathway to personalized medicine 2.0. Int J Colorectal Dis 32: 1077-1084, 2017.

102. Stringer AM, Al-Dasooqi N, Bowen JM, Tan TH, Radzuan M, Logan RM, Mayo B, Keefe DM and Gibson RJ Biomarkers of chemotherapy-induced diarrhoea: A clinical study of intestinal microbiome alterations, inflammation and circulating matrix metalloproteinases. Support Care Cancer 21: 1843-1852, 2013.

103. Lin XB, Dieleman LA, Ketabi A, Bibova I, Sawyer MB, Xue H, Field CJ, Baracos VE and Gänzle MG: Irinotecan (CPT-11) chemotherapy alters intestinal microbiota in tumour bearing rats. PLoS One 7: e39764, 2012.

104. Panebianco C, Adamberg K, Jaagura M, Copetti M, Fontana A, Adamberg S, Kolk K, Vilu R, Andriulli A and Pazienza V: Influence of gemcitabine chemotherapy on the microbiota of pancreatic cancer xenografted mice. Cancer Chemother Pharmacol 81: 773-782, 2018.
105. Meunier A, Nerich V, Fagnoni-Legat C, Richard M, Mazel D, Adotevi O, Bertrand X and Hocquet D: Enhanced emergence of antibiotic-resistant pathogenic bacteria after in vitro induction with cancer chemotherapy drugs. J Antimicrob Chemother 74: $1572-1577,2019$.

106. Zwielehner J, Lassl C, Hippe B, Pointner A, Switzeny OJ, Remely M, Kitzweger E, Ruckser R and Haslberger AG: Changes in human fecal microbiota due to chemotherapy analyzed by TaqMan-PCR, 454 sequencing and PCR-DGGE fingerprinting. PLoS One 6: e28654, 2011.

107. Montassier E, Gastinne T, Vangay P, Al-Ghalith GA, des Varannes SB, Massart S, Moreau P, Potel G, de La Cochetière MF, Batard E and Knights D: Chemotherapydriven dysbiosis in the intestinal microbiome. Aliment Pharmacol Ther 42: 515-528, 2015.

108. Kong C, Gao R, Yan X, Huang L, He J, Li H, You J and Qin H: Alterations in intestinal microbiota of colorectal cancer patients receiving radical surgery combined with adjuvant $\mathrm{CapeOx}$ therapy. Sci China Life Sci 62: 1178-1193, 2019.

109. Lozupone CA, Stombaugh JI, Gordon JI, Jansson JK and Knight R: Diversity, stability and resilience of the human gut microbiota. Nature 489: 220-230, 2012.

110. Bhatt AP, Pellock SJ, Biernat KA, Walton WG, Wallace BD, Creekmore BC, Letertre MM, Swann JR, Wilson ID, Roques JR, et al: Targeted inhibition of gut bacterial $\beta$-glucuronidase activity enhances anticancer drug efficacy. Proc Natl Acad Sci USA 117: 7374-7381, 2020.

111. Roberts AB, Wallace BD, Venkatesh MK, Mani S and Redinbo MR: Molecular insights into microbial $\beta$-glucuronidase inhibition to abrogate CPT-11 toxicity. Mol Pharmacol 84: 208-217, 2013.

112. Pellock SJ, Walton WG, Biernat KA, Torres-Rivera D, Creekmore BC, Xu Y, Liu J, Tripathy A, Stewart LJ and Redinbo MR: Three structurally and functionally distinct $\beta$-glucuronidases from the human gut microbe. J Biol Chem 293: 18559-18573, 2018

113. Wallace BD, Roberts AB, Pollet RM, Ingle JD, Biernat KA, Pellock SJ, Venkatesh MK, Guthrie L, O'Neal SK, Robinson SJ, et al: Structure and inhibition of microbiome $\beta$-glucuronidases essential to the alleviation of cancer drug toxicity. Chem Biol 22: 1238-1249, 2015.

114. Wallace BD, Wang H, Lane KT, Scott JE, Orans J, Koo JS, Venkatesh M, Jobin C, Yeh LA, Mani S and Redinbo MR: Alleviating cancer drug toxicity by inhibiting a bacterial enzyme. Science 330: 831-835, 2010.

115. Chamseddine AN, Ducreux M, Armand JP, Paoletti X, Satar T, Paci A and Mir O: Intestinal bacterial $\beta$-glucuronidase as a possible predictive biomarker of irinotecan-induced diarrhea severity. Pharmacol Ther 199: 1-15, 2019

116. Arumugam M, Raes J, Pelletier E, Paslier DL, Yamada T, Mende DR, Fernandes GR, Tap J, Bruls T, Batto JM, et al: Enterotypes of the human gut microbiome. Nature 473: 174-180, 2011.

117. Dougan SK: The pancreatic cancer microenvironment. Cancer J 23: 321-325, 2017

118. Binenbaum Y, Na'ara S and Gil Z: Gemcitabine resistance in pancreatic ductal adenocarcinoma. Drug Resist Updat 23: $55-68,2015$

119. Halbrook CJ, Pontious C, Kovalenko I, Lapienyte L, Dreyer S, Lee HJ, Thurston G, Zhang Y, Lazarus J, Sajjakulnukit P, et al: Macrophage-released pyrimidines inhibit gemcitabine therapy in pancreatic cancer. Cell Metab 29: 1390-1399, 2019.

120. Alexander JL, Wilson ID, Teare J, Marchesi JR, Nicholson JK and Kinross JM: Gut microbiota modulation of chemotherapy efficacy and toxicity. Nat Rev Gastroenterol Hepatol 14: 356-365, 2017

121. Villéger R, Lopès A, Carrier G, Veziant J, Billard E, Barnich N, Gagnière J, Vazeille $\mathrm{E}$ and Bonnet $\mathrm{M}$ : Intestinal microbiota: A novel target to improve anti-tumor treatment? Int J Mol Sci 20: 4584,2019

122. McQuade JL, Daniel CR, Helmink BA and Wargo JA: Modulating the microbiome to improve therapeutic response in cancer. Lancet Oncol 20: e77-e91, 2019.

123. Becattini S, Taur Y and Pamer EG: Antibiotic-induced changes in the intestinal microbiota and disease. Trends Mol Med 22: 458-478, 2016

124. Flieger D, Klassert C, Hainke S, Keller R, Kleinschmidt R and Fischbach W: Phase II clinical trial for prevention of delayed diarrhea with cholestyramine/levofloxacin in the second-line treatment with irinotecan biweekly in patients with metastatic colorectal carcinoma. Oncology 72: 10-16, 2007. 
125. Kurita A, Kado S, Matsumoto T, Asakawa N, Kaneda N, Kato I, Uchida K, Onoue M and Yokokura T: Streptomycin alleviates irinotecan-induced delayed-onset diarrhea in rats by a mechanism other than inhibition of $\beta$-glucuronidase activity in intestinal lumen. Cancer Chemother Pharmacol 67: 201-213, 2011.

126. Iida N, Mizukoshi E, Yamashita T, Terashima T, Arai K, Seishima $\mathbf{J}$ and Kaneko S: Overuse of antianaerobic drug is associated with poor postchemotherapy prognosis of patients with hepatocellular carcinoma. Int J Cancer 145: 2701-2711, 2019.

127. Levy SB and Marshall B: Antibacterial resistance worldwide: Causes, challenges and responses. Nat Med 10 (12 Suppl): S122-S129, 2004.

128. George Kerry R, Patra JK, Gouda S, Park Y, Shin HS and Das G: Benefaction of probiotics for human health: A review. J Food Drug Anal 26: 927-939, 2018.

129. Yu AQ and Li L: The potential role of probiotics in cancer prevention and treatment. Nutr Cancer 68: 535-544, 2016.

130. Vivarelli S, Salemi R, Candido S, Falzone L, Santagati M, Stefani S, Torino F, Banna GL, Tonini G and Libra M: Gut microbiota and cancer: From pathogenesis to therapy. Cancers (Basel) 11: 38, 2019.

131. An J and Ha EM: Combination therapy of lactobacillus plantarum supernatant and 5-fluouracil increases chemosensitivity in colorectal cancer cells. J Microbiol Biotechnol 26: 1490-1503, 2016

132. Chang CW, Liu CY, Lee HC, Huang YH, Li LH, Chiau JS, Wang TE, Chu CH, Shih SC, Tsai TH and Chen YJ: Lactobacillus casei variety rhamnosus probiotic preventively attenuates 5-fluorouracil/oxaliplatin-induced intestinal injury in a syngeneic colorectal cancer model. Front Microbiol 9: 983, 2018.
133. Wang Y, Sun L, Chen S, Guo S, Yue T, Hou Q, Feng M, Xu H, Liu Y, Wang P and Pan Y: The administration of Escherichia coli Nissle 1917 ameliorates irinotecan-induced intestinal barrier dysfunction and gut microbial dysbiosis in mice. Life Sci 231: 116529, 2019.

134. Serkova MI, Urtenova MA, Tkachenko EI, Avalueva EB, Orlov SV, Ivanov SV, Orishak EA and Skazyvaeva EV: On the possibilities of correction of changes of the gastrointestinal tract microbiota in patients with lung cancer treated receiving chemotherapy. Eksp Klin Gastroenterol: 15-20, 2013.

135. Mego M, Koncekova R, Mikuskova E, Drgona L, Ebringer L, Demitrovicova L, Nemova I, Trupl J, Mardiak J, Koza I and Zajac V: Prevention of febrile neutropenia in cancer patients by probiotic strain Enterococcus faecium M-74. Phase II study. Support Care Cancer 14: 285-290, 2006.

136. Picó-Monllor JA and Mingot-Ascencao JM: Search and selection of probiotics that improve mucositis symptoms in oncologic patients. A systematic review. Nutrients 11: 2322, 2019.

137. González-Sarrías A, Tome-Carneiro J, Bellesia A, Tomás-Barberán FA and Espin JC: The ellagic acid-derived gut microbiota metabolite, urolithin A, potentiates the anticancer effects of 5-fluorouracil chemotherapy on human colon cancer cells. Food Funct 6: 1460-1469, 2015

138. Le Bastard Q, Ward T, Sidiropoulos D, Hillmann BM, Chun CL, Sadowsky MJ, Knights D and Montassier E: Fecal microbiota transplantation reverses antibiotic and chemotherapy-induced gut dysbiosis in mice. Sci Rep 8: 6219, 2018.

This work is licensed under a Creative Commons

Attribution-NonCommercial-NoDerivatives 4.0 International (CC BY-NC-ND 4.0) License. 